\title{
Arsenic trioxide-loaded CalliSpheres: In vitro study of drug release and antitumor activity, and in vivo study of pharmacokinetics, treatment efficacy and safety in liver cancer
}

\author{
XUHUA DUAN*, GUORUI ZHAO*, XINWEI HAN, JIANZHUANG REN, \\ HAO LI, PENGFEI CHEN, MANZHOU WANG and SHUGUANG JU \\ Department of Interventional Radiology, The First Affiliated Hospital of \\ Zhengzhou University, Zhengzhou, Henan 450052, P.R. China
}

Received May 12, 2019; Accepted July 20, 2020

DOI: $10.3892 /$ or.2021.8075

\begin{abstract}
The aim of the present study was to investigate the arsenic trioxide (ATO) loading/releasing efficiency of CalliSphere beads (CBs), as well as the in vitro anticancer activity, in vivo pharmacokinetics, treatment efficacy and safety of ATO-eluting CBs in liver cancer. The ATO loading and releasing efficiencies in CBs were evaluated. Furthermore, cell viability, invasion, apoptosis, VEGF expression and MMP9 expression were determined in liver cancer cells treated with ATO-eluting CBs or ATO solution. Rabbit liver models were established and underwent TACE with ATO-eluting CBs or ATO/lipiodol emulsion. Subsequently, their ATO pharmacokinetics were determined and macroscopic/microscopic examinations were conducted. In vitro, CB-loaded ATO increased during $40 \mathrm{~min}$ with an optimal loading efficiency of $23.0 \pm 2.5 \%$, and released ATO rapidly within the first $30 \mathrm{~min}(31.40 \pm 10.0 \%)$ then slowed down within the latter $48 \mathrm{~h}(47.20 \pm 4.70 \%)$. ATO-eluting CBs exhibited decreased cell viability to some extent and similar invasive cell count, apoptosis rate, VEGF and MMP9 levels compared with ATO solution at various concentrations and time-points. In vivo, ATO concentration was lower in plasma, but higher in tumor tissues, and necrosis was more complete in tumor
\end{abstract}

Correspondence to: Dr Xinwei Han or Dr Jianzhuang Ren, Department of Interventional Radiology, The First Affiliated Hospital of Zhengzhou University, 1 East Jianshe Road, Zhengzhou, Henan 450052, P.R. China

E-mail: hanyuntang813@163.com

E-mail: renfu4073081505@163.com

*Contributed equally

Abbreviations: ATO, arsenic trioxide; CBs, CalliSphere beads; TACE, transhepatic arterial chemoembolization; cTACE, conventional TACE; PBS, phosphate-buffered saline; CCK-8, Cell Counting Kit-8; qPCR, quantitative polymerase chain reaction

Key words: liver cancer, CalliSpheres, arsenic trioxide, loading, release tissue while milder in normal liver parenchyma after rabbit liver was embolized with ATO-eluting CBs compared with ATO/lipiodol emulsion. ATO-eluting CBs may be a novel and promising therapeutic option in treating liver cancer.

\section{Introduction}

Liver cancer is one of the most prevalent types of cancer worldwide, and was the seventh most frequently diagnosed cancer and the second leading cause of cancer-associated deaths in 2018 (1), with 841,000 new cases and 781,000 relevant deaths, accounting for $4.7 \%$ and $8.2 \%$ of all cancer cases and deaths worldwide, respectively (2). Although liver resection has curative potential for patients with early-staged liver cancer, the majority of patients are diagnosed with intermediateto-advanced staged cancer due to the often asymptomatic onset of the disease (3). For patients with unresectable liver cancer, prolonging the survival and maintaining the quality of life is paramount. Transcatheter arterial chemoembolization (TACE) is a locoregional therapy that not only blocks the tumor blood supply through the use of an embolization agent but also infiltrates the tumor tissue resulting in a high concentration of the chemotherapeutic agent specifically within the tumor tissue. TACE is widely utilized to treat patients with unresectable liver cancer due to its favorable efficacy and tolerable side effects (4). Traditionally, the drug carriers in conventional TACE (cTACE) consist of lipiodol, a gelatin sponge, particles and other compounds, which fail to stably release chemotherapeutic agents and embolize target arteries accurately and completely (5). During the past decade, microspheres, a novel drug carrier, have been developed and have become increasingly popular with regard to TACE (6). Compared with conventional drug carriers, microspheres can be used to achieve more sustained drug release, which prolongs the antitumor efficacy of the chemotherapeutic agents $(7,8)$. In addition, microspheres block feeding arteries more accurately and completely than those of conventional drug carriers, leading to improved tumor necrosis effects and reduced chemotherapeutic side effects (9). Due to the advantages of microspheres over conventional drug carriers, microspheres are being increasingly used in TACE. 
In recent years, several microsphere products have become commercially available in China, including CalliSphere beads (CBs) (Jiangsu Hengrui Medicine Co. Ltd.), DC beads (BTG) and HepaSphere (Merit Medical) $(8,10,11)$. Among these, CBs are the first microsphere product independently developed by a local Chinese pharmaceutical company (12). CBs are made of polyvinyl alcohol, which is hydrophilic, nontoxic, biocompatible and nonabsorbable $(12,13)$. Clinical studies have revealed favorable efficiency and safety of CBs in treating patients with liver cancer $(14,15)$. Considering that China has a large number of patients who suffer from liver cancer, accounting for $\sim 50 \%$ of the estimated number of liver cancer patients worldwide, CBs may be used to promising effect in China.

Arsenic trioxide (ATO), an inorganic compound which is known for its hypertoxicity in the ancient world, has been approved for treating acute promyelocytic leukemia (APL) by the American Food and Drug Administration in 2000 due to its anti-APL properties (16). Previously, ATO was demonstrated to exhibit effects on stem cell and metastasis in HCC. For example, a noteworthy study revealed the potent antitumor effects of the combination of ATO and sorafenib through a TRAIL-dependent pathway in Huh7 and freshlyisolated HCC cells (17). Another study revealed that low a dose of ATO was sufficient to suppress vasculogenic mimicry in hepatoblastoma without cell apoptosis (18). Furthermore, ATO has been demonstrated to reduce liver cancer stem cells and metastasis by targeting a serum response factor/minichromosome maintenance protein 7 complex (19). Recently, ATO was also revealed to be efficient for treatment of patients with liver cancer and is used as a palliative treatment in patients with advanced liver cancer in China (20). Considering that systemic chemotherapy of ATO can result in severe adverse events (such as convulsions, cardiovascular problems and kidney failure), in the present study, it was hypothesized that ATO-eluting microspheres for TACE may be a promising strategy to maximize the antitumor activity of ATO while minimizing the systemic toxicity (21). To the best of our knowledge, there are no studies examining the use of ATO-eluting microspheres and assessing their antitumor efficacy. Therefore, the aim of the present study was to investigate the ATO loading and releasing efficiencies of $\mathrm{CBs}$, and to further explore the anticancer activity of ATO-eluting CBs in liver cancer cells and its pharmacokinetics, treatment efficacy as well as safety in a rabbit model of liver cancer.

\section{Materials and methods}

Study design. The present was divided into three parts. In the first part, the ATO loading and releasing efficiencies of CBs were evaluated in vitro. In the second part, comparisons of cell viability, invasion, apoptosis and expression of vascular endothelial growth factor (VEGF) and matrix metalloproteinase 9 (MMP-9) in liver cancer cell lines (HepG2 and MHCC97H) were performed by administrating liver cancer cells with ATO-eluting CBs (CBATO group) or ATO solution (ATO group). In the third part, comparisons of ATO concentrations in plasma, tumor tissue and normal liver parenchyma, as well as macroscopic findings of liver tissue were performed between the CBATO group and the cTACE group in an animal model of liver cancer.
Preparation of CB and ATO solution. CBs (100-300 $\mu \mathrm{m})$ were purchased from Jiangsu Hengrui Medicine Co., Ltd., and ATO was purchased from Shanghai LoyMed Pharma Co., Ltd. The ATO solution $(10 \mathrm{mg} / \mathrm{ml})$ was prepared as follows: $60 \mathrm{mg}$ ATO powder was added to $6 \mathrm{ml}$ glucose solution $(5 \%)$ and then mixed vigorously.

Evaluation of ATO loading efficiency in CBs. A total of $1 \mathrm{~g}$ $\mathrm{CB}$ (without water) was added to the $6 \mathrm{ml}$ ATO solution $(10 \mathrm{mg} / \mathrm{ml})$, and the mixture was vibrated carefully for $1 \mathrm{sec}$; $50 \mu 1$ solution was removed from the mixture every $10 \mathrm{~min}$ for $60 \mathrm{~min}$ after vibration and centrifuged at $6660 \mathrm{x} \mathrm{g}$ for $5 \mathrm{~min}$. Subsequently, $2 \mathrm{ml}$ solution was obtained after the upper layer was diluted 100-fold, and the arsenic concentration was determined using the silver diethyldithiocarbamate method, according to a previously published study (22) followed by spectrophotometry $\left(\mathrm{y}=0.0713 \mathrm{x}-0.013, \mathrm{R}^{2}=0.9985 ; \mathrm{n}=3\right)$, and the concentration of ATO was calculated. The amount of ATO in CBs was equivalent to the total amount subtracted from the solution. The experiment was replicated 3 times and the mean value and standard deviation of ATO concentrations were measured, and an ATO loading curve was drawn.

Evaluation of ATO releasing efficiency in CBs. ATO-eluting CBs were placed on a flow cell of the Pharmacopeia flowthrough apparatus 4 (CE6; Sotax), the pump flow rate was set at $5 \mathrm{ml} / \mathrm{min}$ and the releasing medium was $50 \mathrm{ml} \mathrm{PBS}$ (Gibco; Thermo Fisher Scientific, Inc.). From the releasing medium, $4 \mathrm{ml}$ PBS was obtained after $30 \mathrm{~min}, 1,2,4,8,12$, 24 and $48 \mathrm{~h}$ after the start of release, and the ATO concentration was determined as aforementioned. The experiment was replicated 3 times, the mean value and standard deviation of ATO concentrations were measured, and the ATO releasing curve was drawn.

Cell culture. Human liver cancer cell lines, HepG2 and MHCC97H, (donated by Wuhan Union Hospital of Huazhong University of Science and Technology) were cultured in DMEM (Invitrogen; Thermo Fisher Scientific, Inc.) supplemented with 10\% FBS (Gibco; Thermo Fisher Scientific, Inc.), $100 \mathrm{U} / \mathrm{ml}$ and $100 \mu \mathrm{g} / \mathrm{ml}$ streptomycin (both from North China Pharmaceuticals Co., Ltd.) at $37^{\circ} \mathrm{C}$ in a humidified incubator with $5 \% \mathrm{CO}_{2}$. In vitro experiments were performed under sterile conditions.

Assessment of cell viability. Cell viability was performed using a Cell Counting Kit-8 (CCK-8) assay (Dojindo Molecular Technologies, Inc.). HepG2 cells (authenticated by STR profiling) and MHCC97H cells were seeded into a 96-well plate $\left(2 \times 10^{4}\right.$ cells/well), and $200 \mu 1$ medium containing $0,5,10$, 15 or $20 \mathrm{ng} / \mathrm{ml}$ ( $1 \mathrm{ng} / \mathrm{ml}$ ATO, $0.5 \mu \mathrm{mol} / 1$ ATO) ATO-eluting CBs or ATO solution were added to the wells. Subsequently, CCK-8 reagent was added to each well after $0,12,24,48$ or $72 \mathrm{~h}$, and the plate was incubated for a further $4 \mathrm{~h}$ at $37^{\circ} \mathrm{C}$. The absorbance was measured at $450 \mathrm{~nm}$ with an automatic microplate reader.

Assessment of cell invasion. Cell invasion was determined using a Transwell assay (Corning, Inc.). Briefly, $2 \times 10^{4}$ cells $(100 \mu \mathrm{l})$ in $0.5 \% \mathrm{BSA}$-medium was added to the upper chamber 
of the Transwell insert (A 24-well cell culture plate; a polycarbonate filter membrane with a diameter of $6.5 \mathrm{~mm}$ was at the bottom of the chamber; micropore diameter of $8.0 \mu \mathrm{m}$ ) which had been pre-coated with $50 \mu 1$ Matrigel (BD Biosciences), and $600 \mu \mathrm{l}$ medium containing $10 \%$ FBS was added to the lower chamber. After incubation at $37^{\circ} \mathrm{C}$ for $12 \mathrm{~h}$, ATO-eluting CBs or ATO solution were added to the medium to a final ATO concentration of $0,5,10,15$ or $20 \mathrm{ng} / \mathrm{ml}$, and the cells were incubated for a further $48 \mathrm{~h}$. Subsequently, the cells on the upper surface of the filter were removed by wiping, and the cells which had adhered to the undersurface of the filter were fixed in $4 \%$ of paraformaldehyde for $30 \mathrm{~min}$ at $4{ }^{\circ} \mathrm{C}$, stained with $0.5 \%$ crystal violet (Sigma-Aldrich; Merck KGaA) at $23^{\circ} \mathrm{C}$ for $10 \mathrm{~min}$ and imaged using a light microscope; 3 fields (x100) of view were randomly selected for cell counting, and the mean value of the cell count was calculated.

Assessment of apoptosis. Cell apoptosis was analyzed using an Annexin V/propidium iodide (AV/PI) assay. Briefly, liver cancer cells were seeded in a 6 -well plate $\left(5 \times 10^{5}\right.$ cells/well $)$ and then incubated (at $37^{\circ} \mathrm{C}, 5 \% \mathrm{CO}_{2}$ ) for $24 \mathrm{~h}$. Subsequently, $2 \mathrm{ml}$ medium containing $0,5,10,15$ or $20 \mathrm{ng} / \mathrm{ml}$ ATO-eluting CBs or ATO solution were added to the wells, and incubated (at $37^{\circ} \mathrm{C}, 5 \% \mathrm{CO}_{2}$ ) for a further 24 or $48 \mathrm{~h}$. Following incubation, cells were resuspended in $400 \mu \mathrm{l}$ binding buffer, and $5 \mu \mathrm{l}$ AV-FITC (BD Biosciences) and $5 \mu \mathrm{l}$ PI (BD Biosciences) was added. The mixture was incubated $\left(\sim 2-8^{\circ} \mathrm{C}\right.$ in the dark) for $15 \mathrm{~min}$ and the proportion of apoptotic cells was analyzed using a flow cytometer and software (FlowJo 7.6.1 BD; FlowJo, LLC).

Reverse transcription-quantitative $(R T-q) P C R$. Liver cancer cells were seeded in a 6 -well plate $\left(5 \times 10^{5}\right.$ cells/well), and $2 \mathrm{ml}$ medium containing $0,5,10,15$ or $20 \mathrm{ng} / \mathrm{ml}$ ATO-eluting CBs or ATO solution were added to the wells, and incubated for 24 or $48 \mathrm{~h}$. Following treatment, total RNA was extracted from cells using TRIzol ${ }^{\circledR}$ reagent (Invitrogen; Thermo Fisher Scientific, Inc.) and reverse-transcribed to cDNA using PrimeScript ${ }^{\mathrm{TM}}$ RT reagent kit (Takara Bio, Inc.). qPCR was performed using a QuantiNova SYBR-Green PCR kit (Qiagen GmbH) and the mRNA expression levels of VEGF and MMP-9 were determined using the $2^{-\Delta \Delta \mathrm{Cq}}$ method (23). GAPDH was used as an internal reference. The sequences of the primers used were: VEGF forward, 5'-TGTCTAATGCCCTGGAGCCT-3' and reverse, 5'-GCTTGTCACATCTGCAAGTACG-3'; MMP9 forward, 5'-CGGTTTGGAAACGCAGATGG-3' and reverse, 5'-TGGGTGTAGAGTCTCTCGCT-3'; and GADPH forward, 5'-TGGAAGGACTCATGACCACA-3' and reverse, 5'-TTCAGCTCAGGGATGACCTT-3'.

Western blotting. Liver cancer cells were seeded in a 6-well plate $\left(5 \times 10^{5}\right.$ cells/well), and $2 \mathrm{ml}$ medium containing $0,5,10$, 15 or $20 \mathrm{ng} / \mathrm{ml}$ ATO-eluting CBs or ATO solution were added to the wells, and incubated for 24 or $48 \mathrm{~h}$. Total protein was extracted using RIPA lysis and extraction buffer (Thermo Fisher Scientific, Inc.), and the concentration was determined using a BCA kit (Thermo Fisher Scientific, Inc.). Protein samples $(40 \mu \mathrm{g})$ were loaded on an $8 \%$ SDS-gel, resolved using SDS-PAGE and transferred to polyvinylidene fluoride membranes (EMD Millipore). After blocking (the PVDF membranes were immersed and blocked with the TBST sealant containing $5 \%$ skimmed milk powder and stored at $37^{\circ} \mathrm{C}$ for $2 \mathrm{~h}$ ), the membranes were incubated overnight at $4^{\circ} \mathrm{C}$ with antiVEGF (1:1,000; product code ab32152; Abcam) or anti-MMP-9 (1:1,000; product code ab76003; Abcam) primary antibodies. The dilutions for both the antibodies were performed using $5 \%$ BSA. Subsequently, the membranes were incubated with horseradish peroxidase-conjugated secondary antibody goat anti-rabbit IgG-HRP (1:5,000; product code ab6721; Abcam) at $37^{\circ} \mathrm{C}$ for $2 \mathrm{~h}$. Signals were visualized using Pierce enhanced chemiluminescence Western Blotting Substrate (Thermo Fisher Scientific, Inc.). Anti- $\beta$-actin (1:5,000; product code ab227387; Abcam) was used as the loading control. The ImageJ software (1.8.0; National Institutes of Health) was used for densitometric analysis.

Preparation of animal models with VX2 liver cancer. All the procedures on animals were approved by the Institutional Animal Care and Use Committee at our institution (Ethics and Scientific Trial Committee of the First Affiliated Hospital of Zhengzhou University), and the experiments were performed in accordance with institutional guidelines. Adult male New Zealand white rabbits (water ad libitum and regular diets) $(\mathrm{n}=100$; weighing $3.5-4.1 \mathrm{~kg}$ ) raised at a temperature of $\sim 20-27^{\circ} \mathrm{C}$, a humidity greater than $60 \%$, an airflow value not exceeding $0.2 \mathrm{~m} / \mathrm{sec}$, a ventilation frequency of $\sim 10-20$ times $/ \mathrm{h}$, a 12-h light/dark cycle and an indoor noise controlled within $60 \mathrm{~dB}$, were obtained from Henan Experimental Animal Center and active VX2 tumors were implanted into these rabbits under aseptic conditions. In this study, all VX2 models were transplanted with a single tumor. Then, a CT scan was performed 15 days after surgery in the CBATO, CB, cTACE and control groups. The general procedure of implantation was as follows: Rabbits were anesthetized with a mixture of acepromazine [2.5 mg/kg, intramuscularly (i.m.); Sage Chemical Co., Ltd.] and ketamine hydrochloride $(44 \mathrm{mg} / \mathrm{kg}$, i.m.; Jiuxu Pharmaceutical Co., Ltd.), and the anesthetization was maintained using sodium pentobarbital. Subsequently, the abdomen was shaved and prepared for aseptic procedures, and the left lateral lobe of the liver was exposed after a small incision was made. The fresh VX2 tumor was harvested from carriers, minced and inoculated into the liver. Finally, the abdomen was closed in layers to complete the operation. After the surgery, the rabbits were fed for 14 days to ensure the growth of the VX2 tumors, and then contrast-enhanced computed tomography was used to examine the location and size of the tumor in each rabbit. The eligible rabbits were selected for further experiments. The study was approved from The Scientific Research and Clinical Trial Ethics Committee of the First Affiliated Hospital of Zhengzhou University (No. Scientific research-2017-03).

Treatments. A total of 24 rabbit models were deemed suitable for further use, and were randomly divided into the CBATO $(n=12)$ and cTACE group $(n=12)$. The CBATO group received TACE using ATO-eluting CBs (100-300 $\mu \mathrm{m}$; Jiangsu Hengrui Medicine Co. Ltd.; $0.5 \mathrm{mg} / \mathrm{kg}$ ATO), and the cTACE group received TACE using ATO/lipiodol emulsion $(0.5 \mathrm{mg} / \mathrm{kg}$ ATO). The general TACE procedure was as follows: Rabbits were anesthetized as aforementioned, then the right femoral 

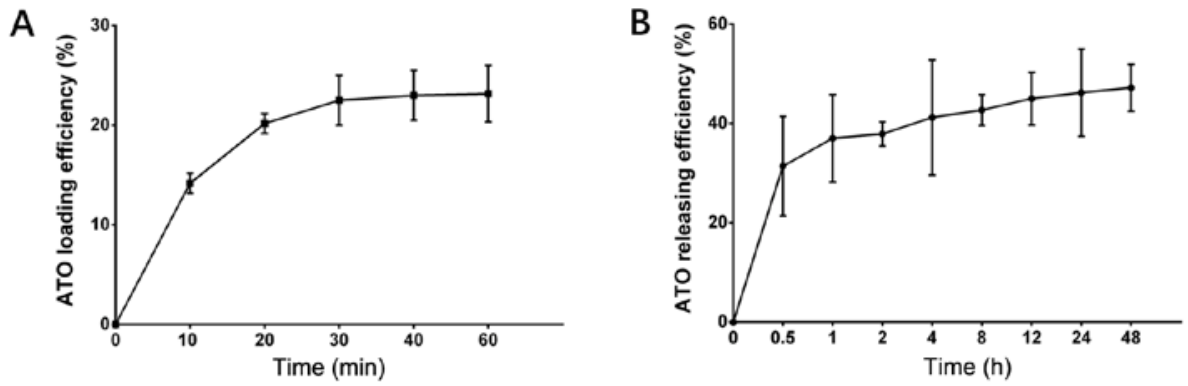

Figure 1. ATO loading and releasing efficiency curves of CBs. (A) The ATO loading efficiency curve of CBs. (B)The ATO releasing efficiency curve of CBs. ATO, arsenic trioxide; CBs, CalliSphere beads.

A

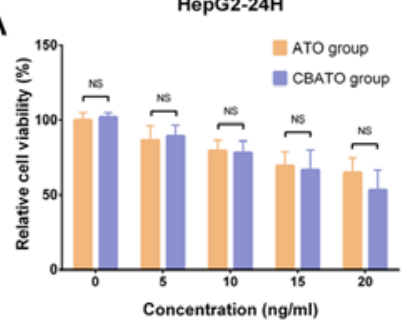

E

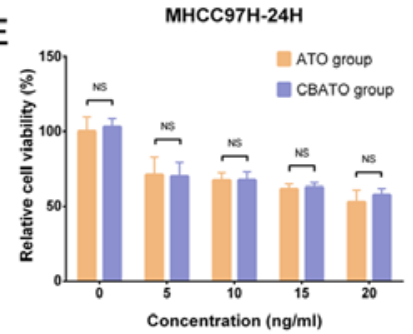

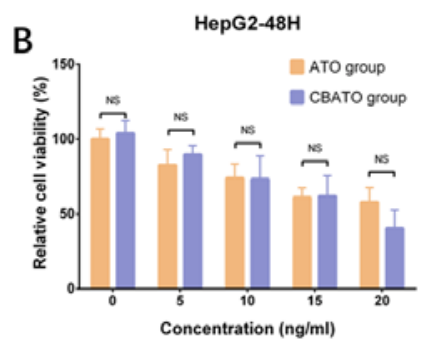

F

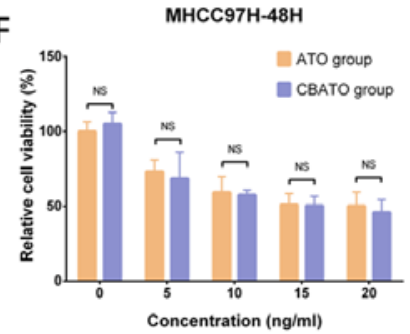

C

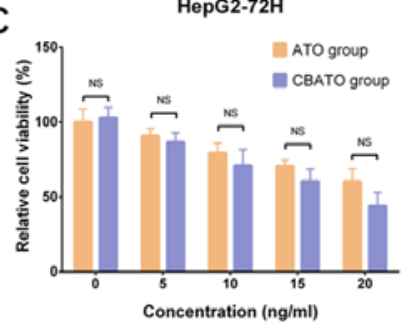

G

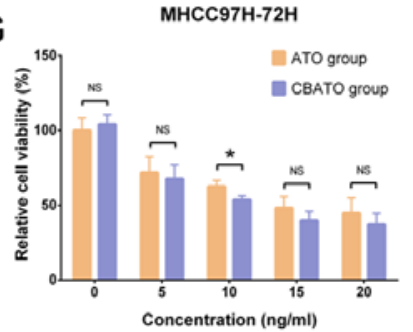

D
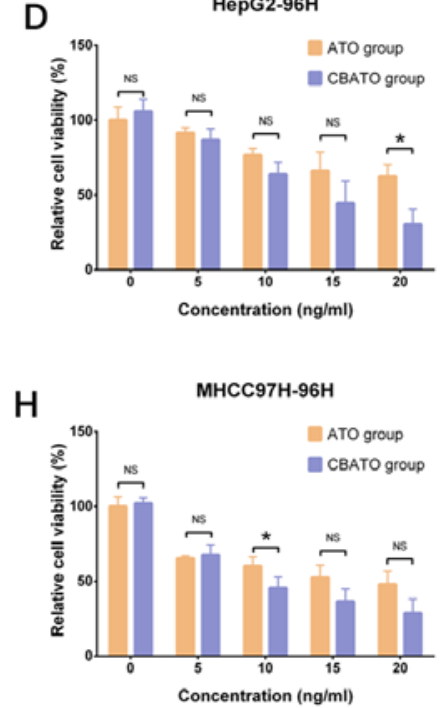

Figure 2. Cell viability in the CBATO and ATO groups in liver cancer cells. Cell viability in HepG2 cells at (A) 24 , (B) 48 , (C) 72 and (D) 96 h between the CBATO group and ATO group. Cell viability in MHCC97H cells at (E) 24, (F) 48, (G) 72 and (H) 96 h between the CBATO and ATO groups. Comparison between the CBATO and ATO groups was determined by t-test, $\mathrm{P}<0.05$ was considered as significant. ${ }^{*} \mathrm{P}<0.05$. Comparison among various concentrations within a group was determined by one-way ANOVA followed by multiple comparisons test (Tukey's test), P<0.05 was considered as significant, the statistical significance was not presented in the figure but described in the Result section. ATO, arsenic trioxide; CBs, CalliSphere beads; NS, no significance.

artery was exposed, and a 4-F sheath (Cook, Inc.) was placed into the artery. Subsequently, a 4-F catheter (Cook, Inc.) was inserted into the hepatic artery to identify its anatomy, and a 2.7-F coaxial microcatheter (Cook, Inc.) was catheterized into the left hepatic artery; the ATO-eluting CBs or ATO/lipiodol emulsion was injected into the target artery. After treatment, the catheters were removed, the artery was ligated, and the rabbits were fed for another 14 days.

Determination of ATO concentration in plasma. Whole blood was obtained after $0.17,0.33,0.67,1,3,6,12,24$ and $72 \mathrm{~h}$ after surgery, and the ATO concentration was determined by liquid chromatography-mass spectrometry.

Gross observation of the liver, histopathological examination of the tumor tissue and determination of ATO concentration in tissues. From both groups, 4 rabbits were euthanized (under deep anesthesia by slow injection of a lethal dose $(100 \mathrm{mg} / \mathrm{kg}$ of body weight of sodium pentobarbital intravenously) after 1,7 or 14 days following the surgery, and gross observation of the liver was performed after 1 or 7 days following the surgery. Subsequently, tumor tissue samples from all rabbits were obtained and dissected into two equal halves. One half was fixed with formalin at $23^{\circ} \mathrm{C}$ for $24 \mathrm{~h}$, mounted on slices and stained with hematoxylin and eosin at $23^{\circ} \mathrm{C}$ for $5 \mathrm{~min}$, and histopathologically examined. The other half was used to determine the ATO concentrations using liquid chromatography-mass spectrometry. Additionally, the normal liver parenchyma was also obtained after euthanasia and the ATO concentration was determined using liquid chromatographymass spectrometry as well.

Euthanasia of the experimental rabbits. According to the American Veterinary Medical Association Guidelines for the Euthanasia of Animals: 2013 Edition (24), in order to reduce the pain of the experimental rabbits, the experimental animals received ear marginal vein rapid injection of sodium pentobarbital $(100 \mathrm{mg} / \mathrm{kg})$, for euthanization; after injection of the drug, the vital signs of the experimental rabbits were checked every 2 min until no heartbeat, no spontaneous breathing for 2-3 min or no blink reflex was observed. In order to decrease the pain and stress, all animals were euthanized under deep anesthesia 
A

ATO

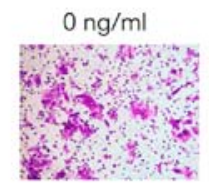

CBATO

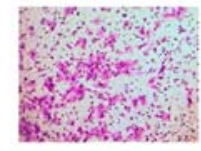

B

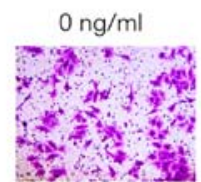

CBATO

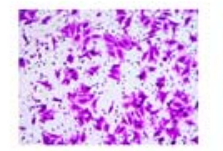

C
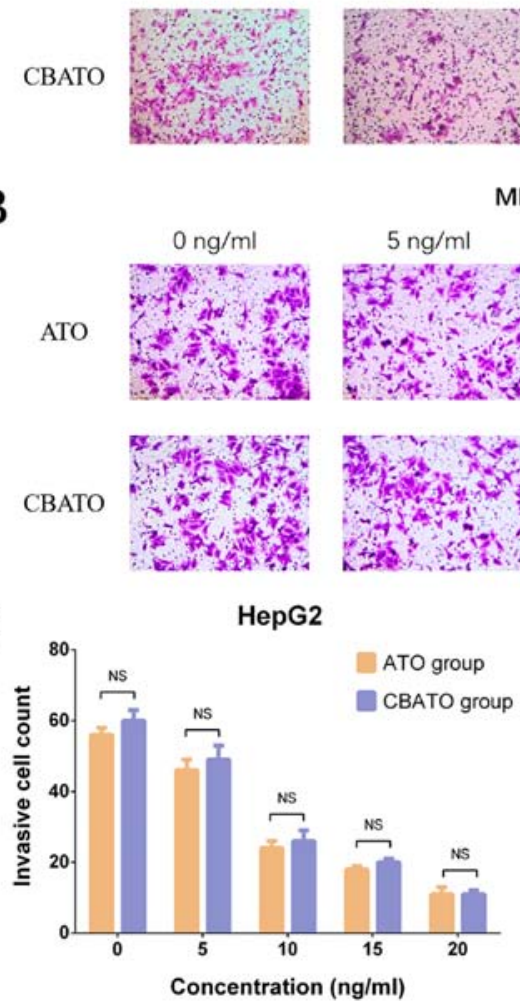

HepG2 cells
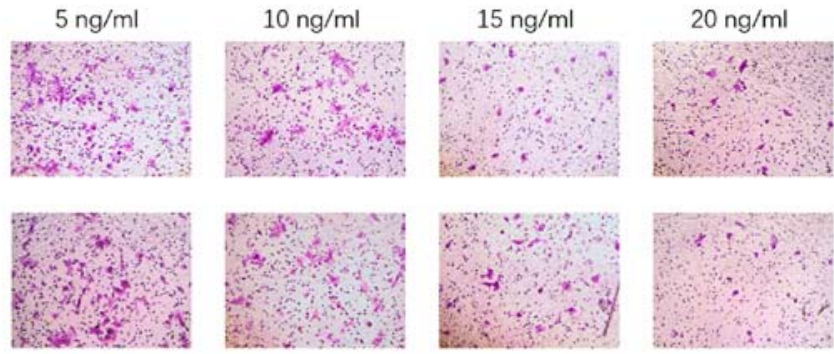

MHCC97H cells
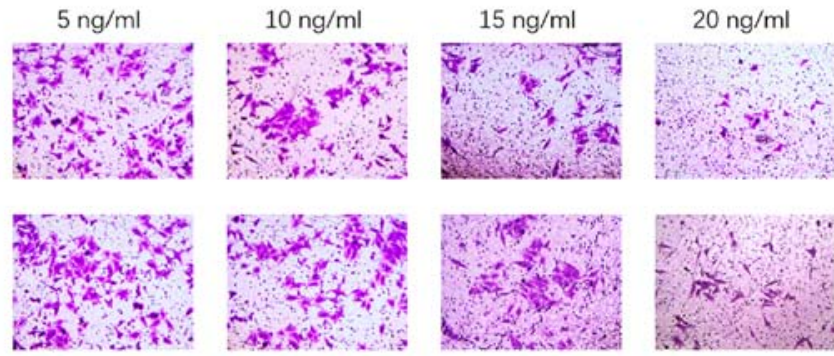

D

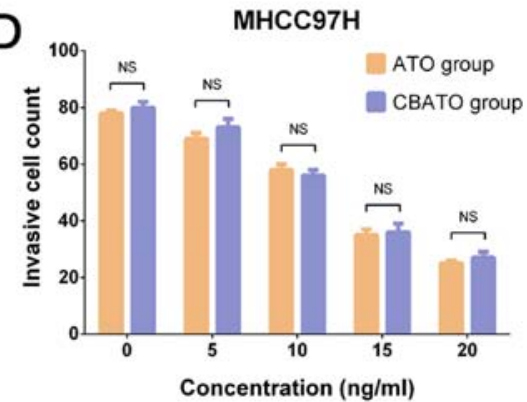

Figure 3. Invasive cell count in the CBATO and ATO groups in liver cancer cells. Invasive cell count in (A and C) HepG2 cells and (B and D) MHCC97H cells between the CBATO and ATO groups. Comparison between the CBATO and ATO groups was determined by t-test, $\mathrm{P}<0.05$ was considered as significant. Comparison among various concentrations within a group was determined by one-way ANOVA followed by multiple comparisons test (Tukey's test), $\mathrm{P}<0.05$ was considered as significant, the statistical significance was not presented in the figure but described in the Result section. ATO, arsenic trioxide; CBs, CalliSphere beads; NS, no significance.

by slow injection of a lethal dose of sodium pentobarbital intravenously, and provided with soft music to terminate their lives in a painless environment. After euthanasia, the rabbits were wrapped and placed on ice temporarily and then harmless treatment was performed. The whole euthanasia process performed was not observed by other experimental rabbits (24).

Statistical analysis. Data are presented as the mean \pm standard deviation. Comparisons between two groups were determined using an unpaired Student's t-test, and comparisons between different concentrations within a group were performed using a one-way ANOVA followed by a post hoc Tukey's test. Statistical analysis was performed in SPSS version 13.0 (SPSS, Inc.), and GraphPad Prism version 6.0 (GraphPad Software, Inc.) was used to plot the graphs. $\mathrm{P}<0.05$ was considered to indicate a statistically significant difference.

\section{Results}

ATO loading and releasing efficiencies of $C B$ s. The amount of ATO loaded by CBs increased with time within the first
$40 \mathrm{~min}$, and the optimal loading efficiency was $23.0 \pm 2.5 \%$ (Fig. 1A). In addition, the ATO release curve revealed that CBs rapidly released substantial ATO during the first 30 min (up to $31.40 \pm 10.0 \%$ ), then slowly released ATO within the latter $48 \mathrm{~h}(47.20 \pm 4.70 \%)$ (Fig. 1B). These data indicated that ATO loading and releasing efficiencies of CBs were acceptable.

Comparison of cell viability between the CBATO group and ATO group in liver cancer cells. To evaluate the anti-liver cancer activities of ATO-eluting CBs, cell viability between the CBATO group and the ATO group was compared. Cell viability was suppressed by ATO in a dose-dependent manner both in the CBATO group (all $\mathrm{P}<0.05$ ) and ATO group (all $\mathrm{P}<0.05$ ) at each time-point in HepG2 cells (Fig. 2A-D) and in MHCC97H cells (Fig. 2E-H). For comparison of cell viability between the CBATO group and the ATO group, there was no difference between the two groups after $24 \mathrm{~h}(\mathrm{P}>0.05$; Fig. 2A), 48 h (P>0.05; Fig. 2B) or 72 h ( $>>0.05$; Fig. 2C) at each concentration, but cell viability was decreased in the CBATO group after $96 \mathrm{~h}(\mathrm{P}<0.05$; Fig. 2D) compared with the ATO group at an ATO concentration of $20 \mathrm{ng} / \mathrm{ml}$ in the HepG2 cells. The cell viability in MHCC97H cells was similar 
A

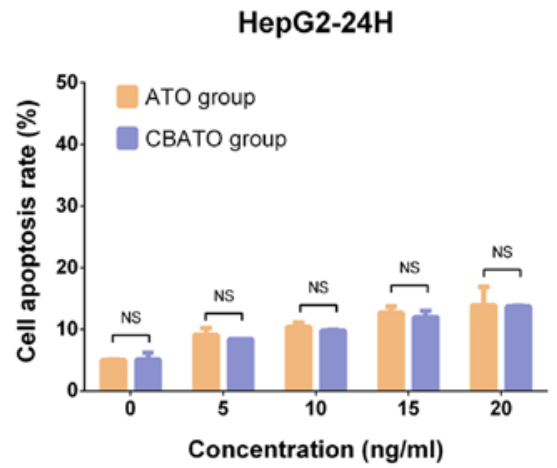

C

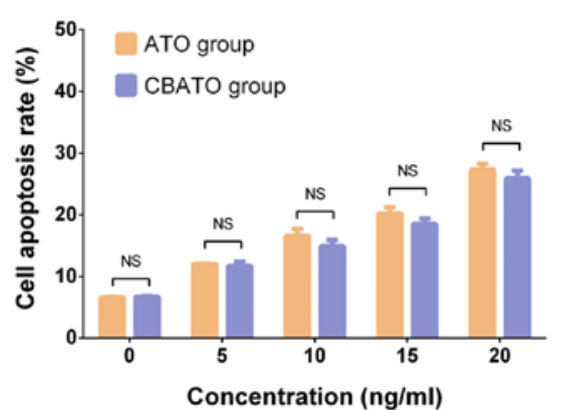

B

HepG2-48H

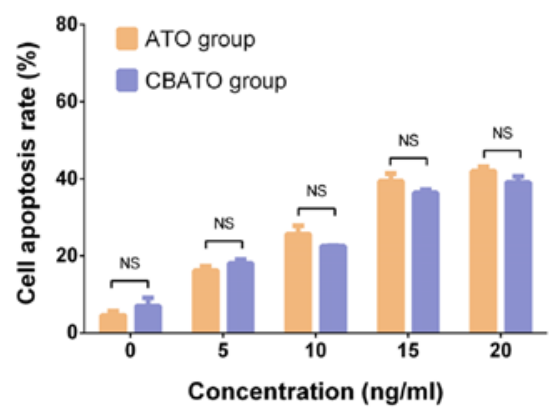

D

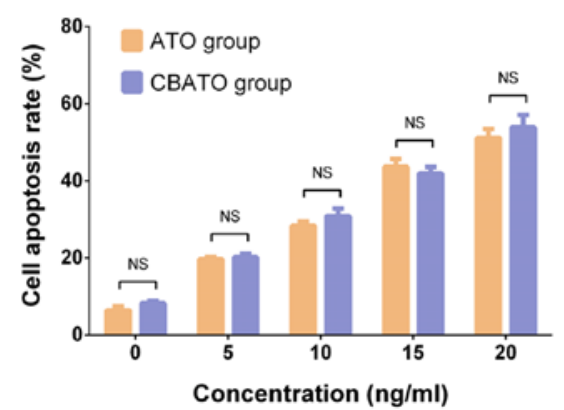

Figure 4. Cell apoptosis rate in the CBATO and ATO groups in liver cancer cells. Cell apoptosis rate in HepG2 cells at (A) $24 \mathrm{~h}$ and (B) $48 \mathrm{~h}$ between the CBATO and ATO groups. Cell apoptosis rate in MHCC97H cells at (C) $24 \mathrm{~h}$ and (D) $48 \mathrm{~h}$ between the CBATO and ATO groups. Comparison between the CBATO and ATO groups was determined by $\mathrm{t}$-test, $\mathrm{P}<0.05$ was considered as significant. Comparison among various concentrations within a group was determined by one-way ANOVA followed by multiple comparisons test (Tukey's test), $\mathrm{P}<0.05$ was considered as significant, the statistical significance was not presented in the figure but described in the Result section. ATO, arsenic trioxide; CBs, CalliSphere beads; NS, no significance.

between the two groups after $24 \mathrm{~h}(\mathrm{P}>0.05$; Fig. $2 \mathrm{E})$ and $48 \mathrm{~h}$ $(\mathrm{P}>0.05$; Fig. 2F) at each concentration, whereas after $72 \mathrm{~h}$ $(\mathrm{P}<0.05$; Fig. 2G) and $96 \mathrm{~h}(\mathrm{P}<0.05$; Fig. $2 \mathrm{H})$, viability in the CBATO group was reduced compared with the ATO group at an ATO concentration of $10 \mathrm{ng} / \mathrm{ml}$. These data indicated that, to some extent, ATO-eluting CBs suppressed the cell viability compared with ATO solution in liver cancer cells.

Comparison of invasion between the CBATO group and ATO group in liver cancer cells. In HepG2 (Fig. 3A and C) and MHCC97H (Fig. 3B and D) cells, invasion was reduced by ATO in a dose-independent manner in both the CBATO group $(\mathrm{P}<0.05)$ and the ATO group $(\mathrm{P}<0.05)$ after $48 \mathrm{~h}$ compared with the control. There was no difference in invasion between the CBATO group and the ATO group at each concentration (all P>0.05; Fig. 3C and D), indicating that ATO-eluting CBs did not affect cell invasion compared with ATO solution in liver cancer cells.

Comparison of cell apoptosis between the CBATO group and ATO group in liver cancer cells. In HepG2 (all $\mathrm{P}<0.05$; Fig. 4A and B) and MHCC97H (all P<0.05; Fig. 4C and D) cells, the cell apoptosis rate was increased by ATO in a dosedependent manner both in the CBATO group and the ATO group after 24 and $48 \mathrm{~h}$. The apoptosis rate was similar between the CBATO group and the ATO group at each concentration after $24 \mathrm{~h}$ (all $\mathrm{P}>0.05$ ) and $48 \mathrm{~h}$ (all $\mathrm{P}>0.05$ ), indicating that ATO-eluting CBs did not affect cell apoptosis compared with ATO solution in liver cancer cells.
Comparison of VEGF $\mathrm{mRNA}$ and protein expression levels between the CBATO group and ATO group in liver cancer cells. As VEGF is reported to serve an important role in promoting growth and invasion of liver cancer cells, the VEGF mRNA and protein expression levels between the CBATO group and TACE group were measured to assess the impact of ATO-eluting CBs on liver cancer cell growth and invasion $(25,26)$. The results revealed that VEGF mRNA and protein expression levels were decreased by ATO in a dosedependent manner in the CBATO group (all $\mathrm{P}<0.05)$ and ATO group (all $\mathrm{P}<0.05)$ after $24 \mathrm{~h}($ Fig. 5A-C) and $48 \mathrm{~h}($ Fig. 5D-F) in HepG2 cells. There was no difference in VEGF mRNA or protein expression levels between the two groups after 24 or $48 \mathrm{~h}$ in HepG2 cells (all P>0.05). In MHCC97H cells, VEGF expression also decreased in a similar manner between the two groups compared with HepG2 cells (Fig. 5G-L). These data indicated that ATO inhibited VEGF expression in liver cancer cells, while ATO-eluting CBs had no impact on the inhibitory effect of ATO.

Comparison of MMP9 $\mathrm{mRNA}$ and protein expression levels between the CBATO group and ATO group in liver cancer cells. MMP9 expression levels were compared between the CBATO group and the TACE group, and the results revealed that MMP9 mRNA and protein expression levels were decreased in an ATO dose-dependent manner both in the CBATO group (all $\mathrm{P}<0.05)$ and ATO group (all $\mathrm{P}<0.05$ ) after $24 \mathrm{~h}$ (Fig. 6A-C) and $48 \mathrm{~h}$ (Fig. 6D-F) in HepG2 cells. However, there was no difference in MMP9 mRNA or protein 
HepG2 cells

A

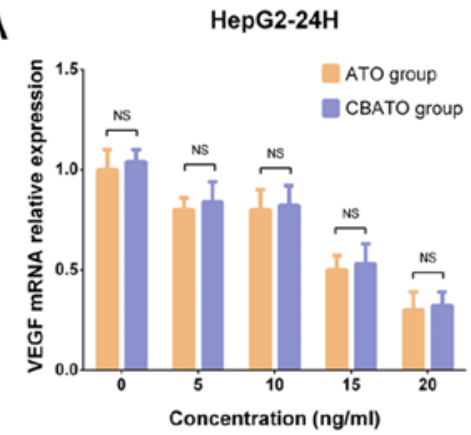

D

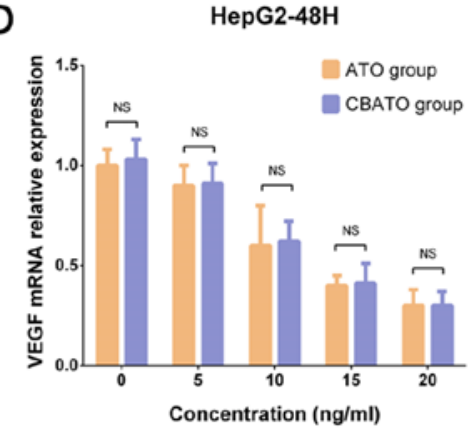

G

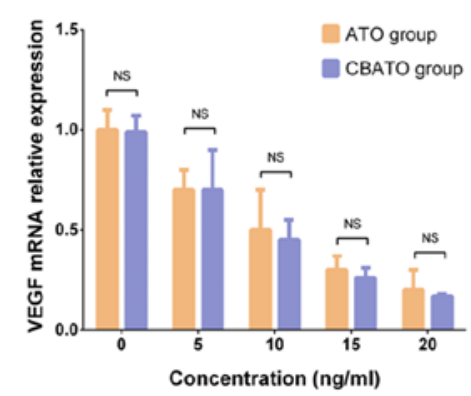

J

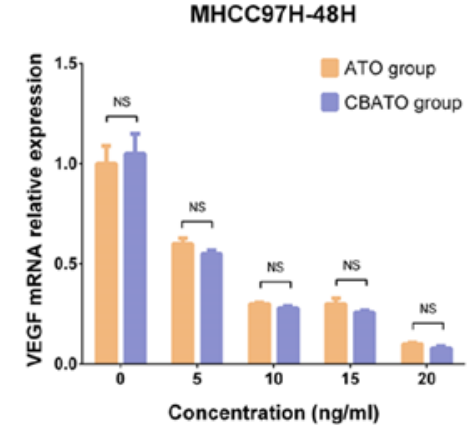

B

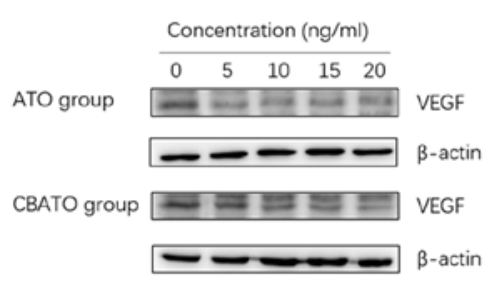

E

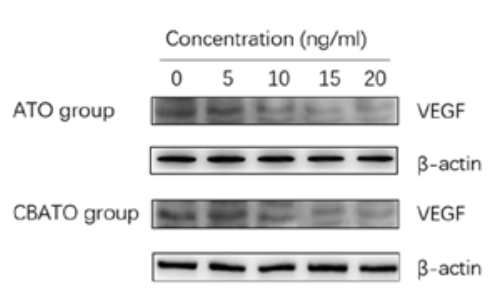

C HepG2-24H

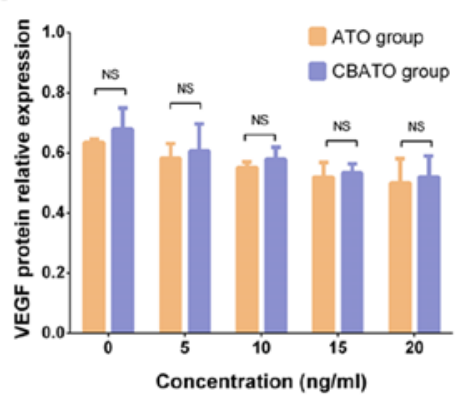

$\mathbf{F}$

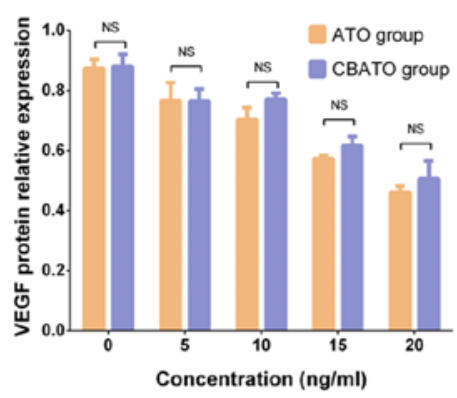

\section{MHCC97H cells}

$\mathrm{H}$

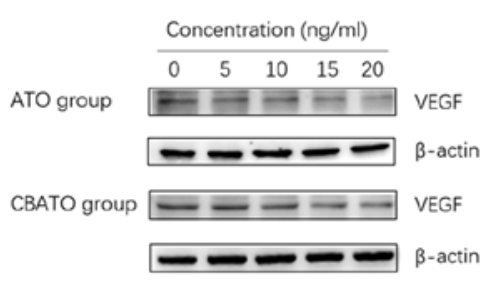

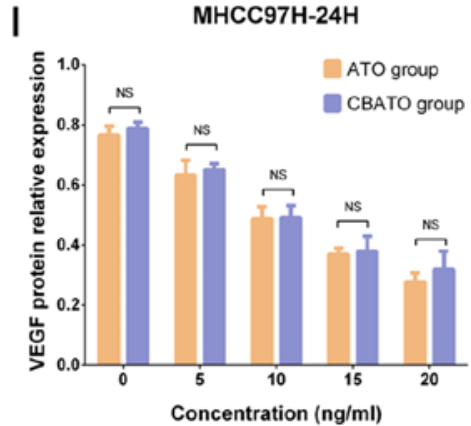

L

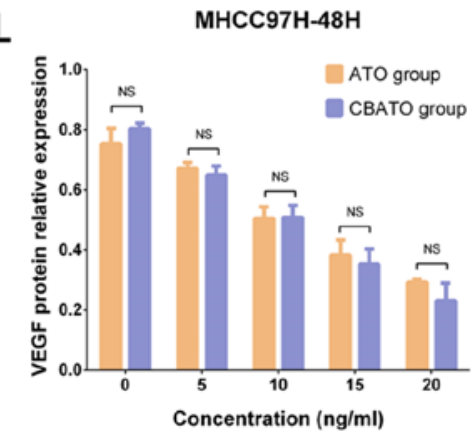

Figure 5. VEGF expression in the CBATO and ATO groups in liver cancer cells. VEGF mRNA expression in HepG2 cells at (A) $24 \mathrm{~h}$ and (D) $48 \mathrm{~h}$ between CBATO and ATO groups. VEGF protein expression in HepG2 cells at (B and C) $24 \mathrm{~h}$ and (E and F) $48 \mathrm{~h}$ between the CBATO and ATO groups. VEGF mRNA expression in MHCC97H cells at (G) $24 \mathrm{~h}$ and (J) $48 \mathrm{~h}$ between CBATO and ATO groups. VEGF protein expression in MHCC97H cells at (H and I) $24 \mathrm{~h}$ and (K and L) $48 \mathrm{~h}$ between the CBATO and ATO groups. Comparison between the CBATO and ATO groups was determined by t-test, P<0.05 was considered as significant. Comparison among various concentrations within a group was determined by one-way ANOVA followed by multiple comparisons test (Tukey's test), $\mathrm{P}<0.05$ was considered as significant, the statistical significance was not presented in the figure but described in the Result section. VEGF, vascular endothelial growth factor; ATO, arsenic trioxide; CBs, CalliSphere beads; NS, no significance.

expression levels observed between the two groups after 24 or $48 \mathrm{~h}$ in HepG2 cells (all P>0.05). In MHCC97H cells,
MMP9 expression was reduced in a similar manner between the two groups compared with HepG2 cells (Fig. 6G-L). These 


\section{HepG2 cells}

A

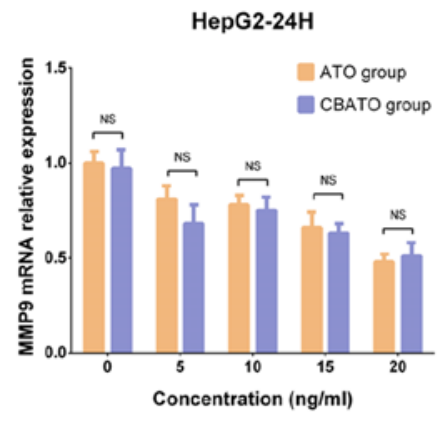

D

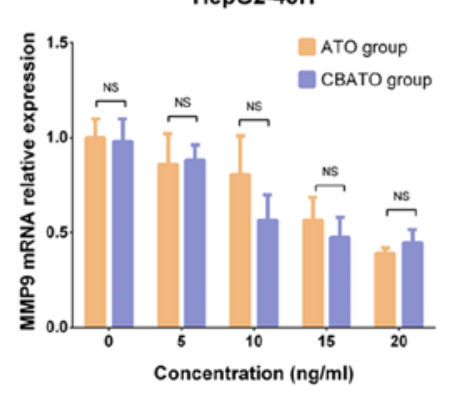

G

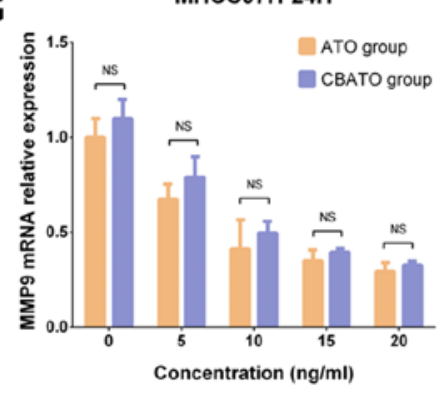

J

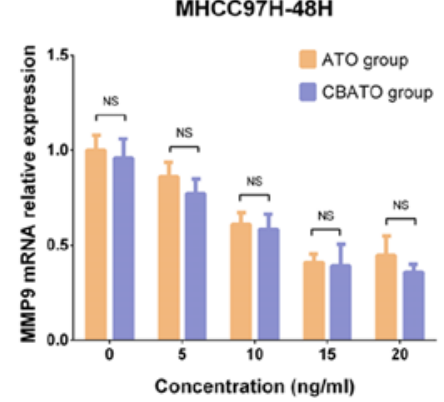

B

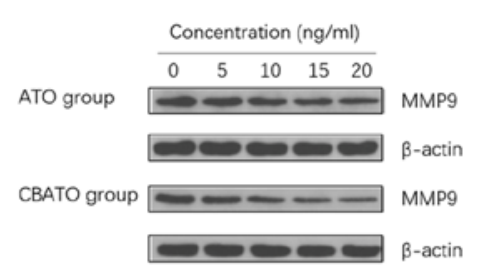

E

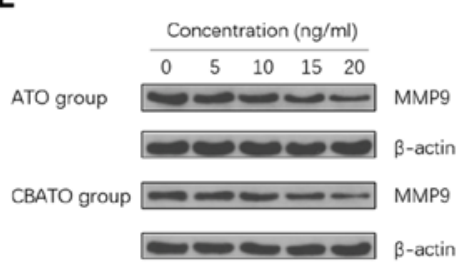

C

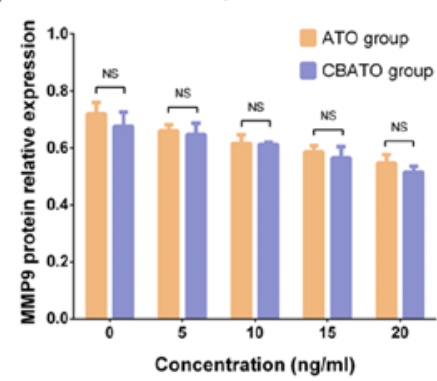

HepG2-48H

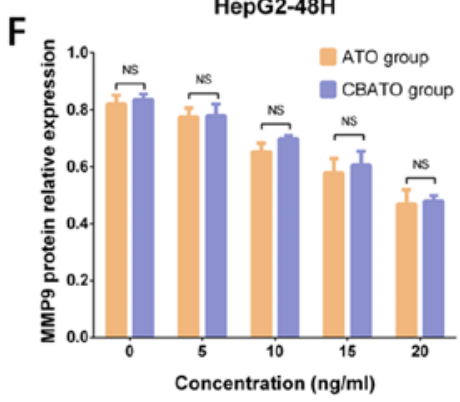

MHCC97H cells

$\mathrm{H} \quad$ MHCC97H-24H I

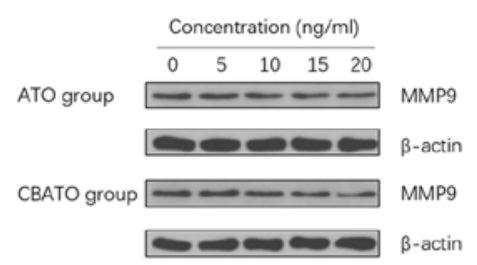

$\mathrm{K}$

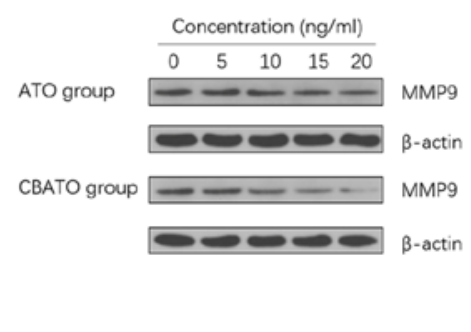

MHCC97H-24H

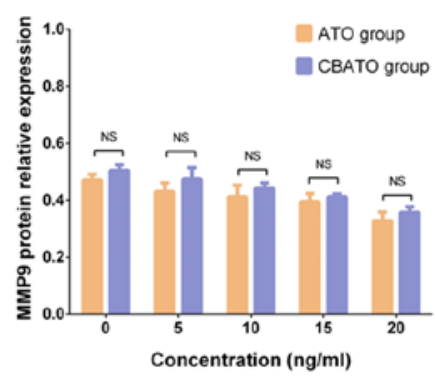

$\mathrm{L}$

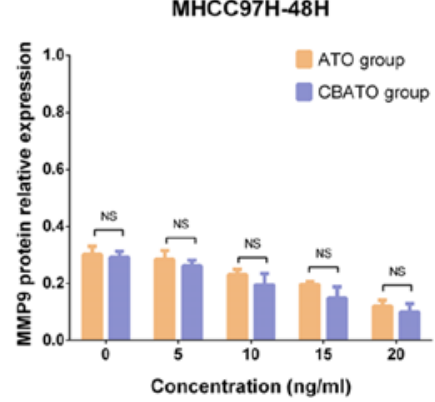

Figure 6. MMP9 expressions in CBATO group and ATO group in liver cancer cells. MMP9 mRNA expression in HepG2 cells at (A) $24 \mathrm{~h}$ and (D) $48 \mathrm{~h}$ between the CBATO and ATO groups. MMP9 protein expression in HepG2 cells at (B and C) $24 \mathrm{~h}$ and (E and F) $48 \mathrm{~h}$ between the CBATO and ATO groups. MMP9 mRNA expression in MHCC97H cells at (G) $24 \mathrm{~h}$ and (J) $48 \mathrm{~h}$ between the CBATO and ATO groups. MMP9 protein expression in MHCC97H cells at (H and $\mathrm{I}) 24 \mathrm{~h}$ and $(\mathrm{K}$ and $\mathrm{L}) 48 \mathrm{~h}$ between the CBATO and ATO groups. Comparison between the CBATO and ATO groups was determined by t-test, $\mathrm{P}<0.05$ was considered as significant. Comparison among various concentrations within a group was determined by one-way ANOVA followed by multiple comparisons test (Tukey's test), $\mathrm{P}<0.05$ was considered as significant, the statistical significance was not presented in the figure but described in the Result section. MMP9, metalloprotease 9; ATO, arsenic trioxide; CBs, CalliSphere beads; NS, no significance.

results indicated that ATO suppressed MMP9 expression in liver cancer cells, while ATO-eluting CBs did not affect the suppressive effect.

Comparison of ATO concentrations in plasma, tumor tissue and normal liver parenchyma between the CBATO group and
cTACE group. ATO concentration in the plasma was higher in the CBATO group compared with the cTACE group after $0.17 \mathrm{~h}(\mathrm{P}<0.001), 0.33 \mathrm{~h}(\mathrm{P}<0.001)$ and $1 \mathrm{~h}(\mathrm{P}<0.05)$ after surgery (Fig. 7A). ATO concentration in the tumor tissue was lower after 1 day $(\mathrm{P}<0.01)$ but higher after days $7(\mathrm{P}<0.001)$ and $14(\mathrm{P}<0.05)$ in the CBATO group compared with the 

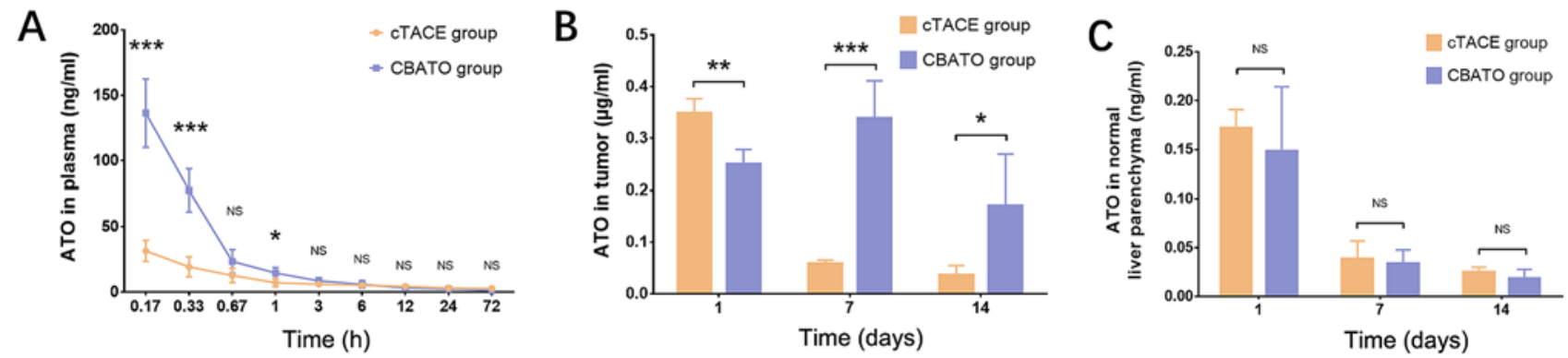

Figure 7. Plasma, tumor tissue and normal liver parenchyma ATO concentrations in the CBATO and cTACE groups. (A) ATO concentration in plasma between the CBATO group and CTACE group. (B) ATO concentration in tumor tissue between the CBATO and cTACE groups. (C) ATO concentration in normal liver parenchyma between the CBATO and cTACE groups. Comparison between the CBATO group and cTACE group was determined by t-test $\mathrm{P}<0.05$ was considered as significant. ${ }^{*} \mathrm{P}<0.05,{ }^{* *} \mathrm{P}<0.01,{ }^{* * *} \mathrm{P}<0.001$. ATO, arsenic trioxide; CBs, CalliSphere beads; cTACE, conventional transcatheter arterial chemoembolization; NS, not significant.

A CTACE group (1 day after the operation)

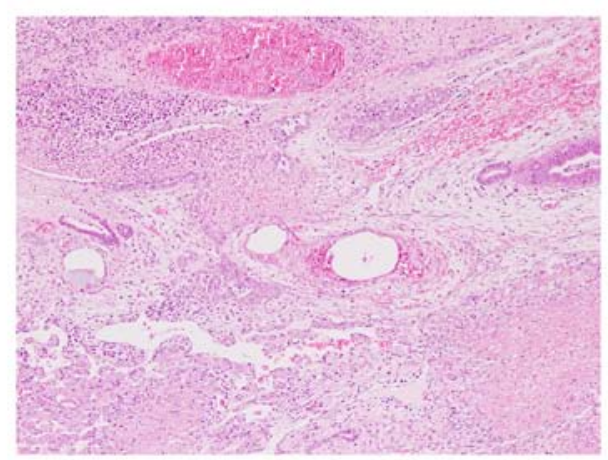

C CBATO group (1 day after the operation)

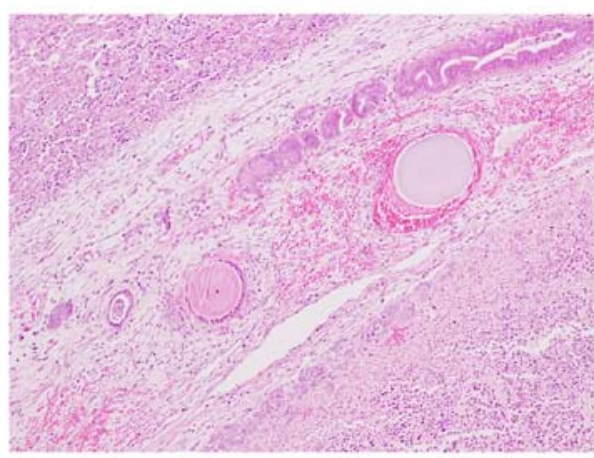

B CTACE group (7 days after the operation)

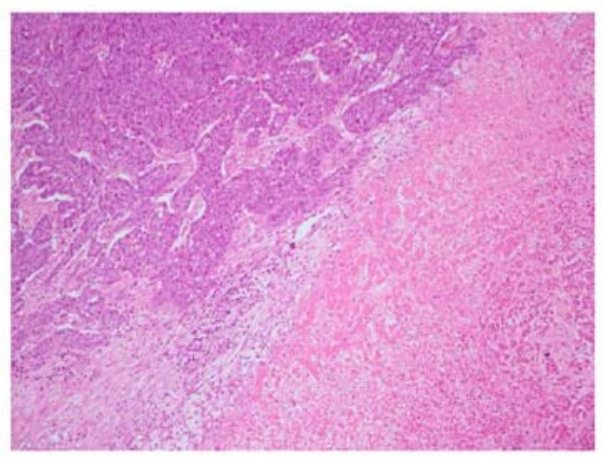

D CBATO group (7 days after the operation)

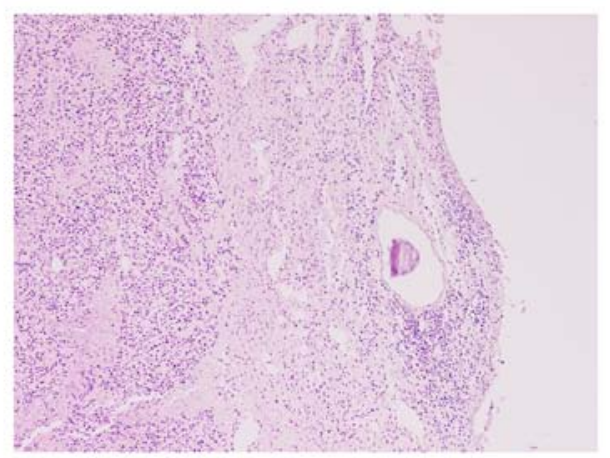

Figure 8. Microscopic findings on tumor tissue in the CBATO and cTACE groups. Histopathologic finds in the cTACE group on the (A) 1st day and (B) 7th day after the surgery. Histopathologic finds in the CBATO group on the (C) 1st day and (D) 7th day after the surgery. ATO, arsenic trioxide; CBs, CalliSphere beads; cTACE, conventional transcatheter arterial chemoembolization

cTACE group (Fig. 7B). The ATO concentration in the normal liver parenchyma was similar between the two groups after $1(\mathrm{P}>0.05), 7(\mathrm{P}>0.05)$ and 14 days $(\mathrm{P}>0.05)$ after the surgery (Fig. 7C). These data indicated that ATO-eluting CBs increased the ATO plasma concentration whilst maximizing ATO tumor concentration compared with the ATO/lipiodol emulsion when using TACE in a rabbit model of liver cancer.

Comparison of histopathological findings in tumor tissue between the CBATO group and cTACE group. Following the surgery, on the $1^{\text {st }}$ day, thrombosis in the vascular lumen, a substantial number of erythrocytes in the vascular wall and necrotic cancer cells in the perivascular areas were observed both in the cTACE group (Fig. 8A) and the CBATO group (Fig. 8C). After the $7^{\text {th }}$ day, there was substantial increase in new cancer cells around the necrotic cancer cells in the cTACE group (Fig. 8B); whereas there were a large number of necrotic cancer cells and inflammatory cells in the perivascular areas in the CBATO group, and no new cancer cells were observed (Fig. 8D), indicating that ATO-eluting CBs had better treatment efficacy compared with ATO/lipiodol emulsion in the TACE treatment of liver cancer.

Comparison of macroscopic findings on rabbit liver between the CBATO group and cTACE group. Following the surgery, 
A

cTACE group (1 day after the operation)

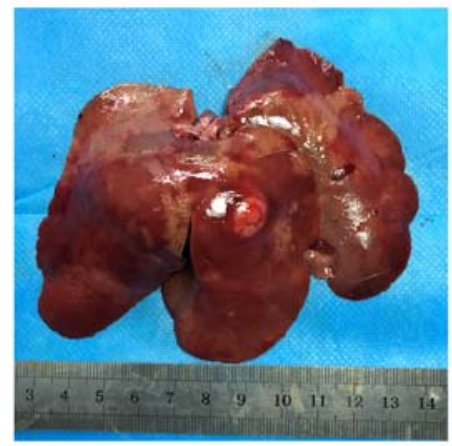

C

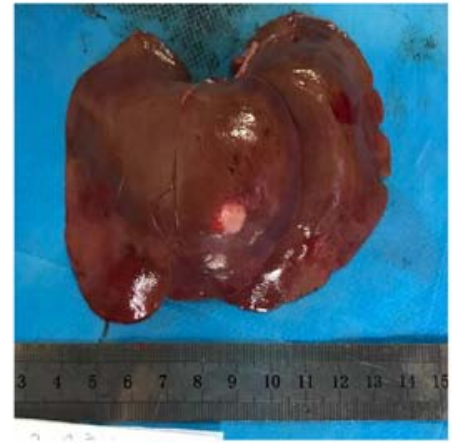

B

cTACE group (7 days after the operation)

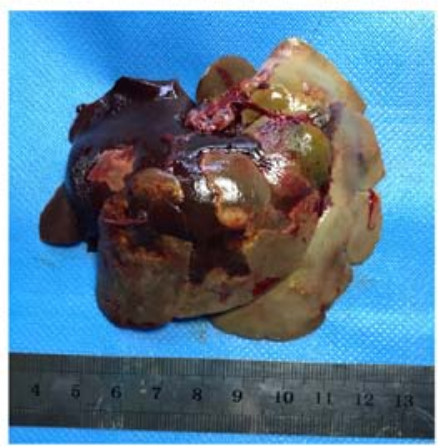

D CBATO group (7 days after the operation)

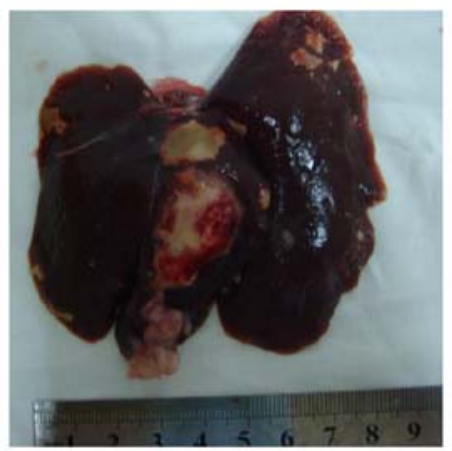

Figure 9. Gross observations on normal liver parenchyma in the CBATO and cTACE groups. Gross observations on normal liver parenchyma in the cTACE group on the (A) 1st day and (B) 7th day after the surgery. Gross observations on normal liver parenchyma in the CBATO group on the (C) 1st day and (D) 7th day after the surgery. ATO, arsenic trioxide; CBs, CalliSphere beads; cTACE, conventional transcatheter arterial chemoembolization.

on the 1st day, ATO-eluting CBs (Fig. 9C) had a greater effect on reduction of extensive necrosis and fibrosis radiologically compared with cTACE (Fig. 9A). After the 7th day, ATO-eluting CBs (Fig. 9D) had less effect on necrosis of tumor tissues and normal liver parenchyma compared with cTACE (Fig. 9B), indicating that ATO-eluting CBs reduced the toxicity on normal liver parenchyma compared with the ATO/lipiodol emulsion. In this study, all VX2 models were transplanted with a single tumor. Then, a CT scan was performed 15 days after surgery in the CBATO, CB, cTACE and control groups, and the tumor maximum length diameter was $10.6 \pm 0.93$, $11.0 \pm 0.78,10.7 \pm 0.71$ and $10.8 \pm 0.85 \mathrm{~mm}$, respectively.

\section{Discussion}

Drug-eluting bead-TACE (DEB-TACE) has been extensively used as an interventional therapy for patients with unresectable liver cancer in recent years owing to its favorable treatment efficacy and moderate adverse events (27). In DEB-TACE, microspheres are reported to be superior to conventional drug carriers/embolization agents (such as lipiodol). For instance, a meta-analysis revealed that microspheres provided improved tumor response, survival and fewer adverse events compared with lipiodol in the treatment of advanced liver cancer patients (28). In addition, several animal studies have demonstrated that microspheres block tumor blood supply more completely and release chemotherapeutic agents more consistently compared with lipiodol, which results in a lower concentration of chemotherapeutic agents in the plasma and normal tissues, and a higher concentration in tumor tissue; this subsequently results in improved antitumoral efficacy and reduced adverse events $(7,8)$. Due to the favorable efficacy and safety of microspheres such as $\mathrm{CBs}$, they are increasingly being used in DEB-TACEmediated treatment of liver cancer.

ATO is an effective chemotherapeutic agent which exhibits powerful cytotoxicity in liver cancer cells via multiple pathways. For example, a previous study revealed that ATO stimulates cell cycle arrest in liver cancer cells (HepG2 and SMMC7721 cell lines) via directly targeting a tumor suppressor gene phosphatase and tensin homologue deleted on chromosome 10 (29). Additionally, ATO was revealed to substantially increase reactive oxygen species (ROS) levels, resulting in cell apoptosis in liver cancer cells (HepG2), and suppressing the ROS levels reversed the apoptosis caused by ATO (30). ATO was also revealed to inhibit liver cancer tumorigenesis via suppression of VEGF and metalloprotease expression (31-33). These studies confirm the favorable anti-liver cancer properties of ATO. However, the clinical application of ATO in treating liver cancer patients has been strictly restricted due to its severe systemic toxicity. In view that DEB-TACE reduces the circulating concentration while increasing the intratumoral concentration of chemotherapeutic drugs, it is hypothesized that ATO-eluting microspheres may enhance the efficiency and tolerance of ATO. However, information concerning the feasibility, treatment efficacy and safety of ATO-eluting microspheres in DEB-TACE treatment of liver cancer is limited. 
In the present study, the in vitro ATO loading and releasing efficiencies of CBs (100-300 $\mu \mathrm{m})$ was evaluated, and it was revealed that ATO was loaded by CBs in a sustainable manner with an optimal loading efficiency of $23.0 \pm 2.5 \%$, which was relatively lower compared with two previous studies. In a previous study, both doxorubicin and irinotecan achieved loading within $40 \mathrm{~min}$ using DC beads (500-700 $\mu \mathrm{m})$ and HepaSphere (400-600 $\mu \mathrm{m})$ (34). In another study, $>99 \%$ of doxorubicin was loaded within $1 \mathrm{~h}$ using DC beads (100$300 \mu \mathrm{m})$, LifePearl $(200 \mu \mathrm{m})$, HepaSphere $(30-60 \mu \mathrm{m})$ and Tandem (100 $\mu \mathrm{m})$ (35). Possible reasons for the discrepancy may be: i) The previous studies utilized doxorubicin or irinotecan as a loading agent, which may form strong ionic interaction with microspheres. The interaction between ATO and microspheres may be weaker compared with the interaction between doxorubicin or irinotecan and microspheres, thereby resulting in a lower loading efficiency. ii) Microspheres are made of different materials (such as sulfonate-modified polyvinyl alcohol or sodium acrylate-modified polyvinyl alcohol), which may influence the interaction between ATO and microspheres, as well. iii) Different solutions (such as saline and deionized water) may also affect the loading efficiency. In the present study, it was observed that CBs released ATO in a rapid manner within the first $30 \mathrm{~min}(31.40 \pm 10.0 \%)$ then released ATO in a prolonged manner for the following $48 \mathrm{~h}(47.20 \pm 4.70 \%)$, which was also relatively different from previous studies, possibly due to the aforementioned reasons. In brief, the present study revealed that ATO could be loaded and released by $\mathrm{CBs}$ favorably.

As ATO exhibits favorable efficacy in liver cancer patients, and CBs exhibited acceptable ATO loading and releasing efficiencies, it is hypothesized that ATO-eluting CBs has a good efficacy for treating liver cancer. However, the in vitro anti-liver cancer activity of ATO-eluting CBs has not been demonstrated until now, to the best of our knowledge. Therefore, cell viability, the apoptosis rate and invasive cell count were compared by treating liver cancer cells with ATO-eluting CBs or ATO solution, and it was revealed that ATO-eluting CBs reduced cell viability to some extent and did not affect invasive cell count or the apoptosis rate compared with ATO solution in liver cancer cells. Additionally, liver cancer cells treated with ATO-eluting CBs or ATO solution exhibited downregulated VEGF and MMP9 expression to a similar degree, both of which are reported to promote cell invasion $(25,26)$. The results of the present study indicated that ATO loaded by CBs exhibited slightly improved efficacy in suppressing liver cancer progression compared with the ATO solution, which may be due to the fact that $\mathrm{CBs}$ surrounded the liver cancer cells, which caused a large ATO concentration gradient between the extra-cell matrix and cytoplasm, thereby facilitating the diffusion of ATO into liver cancer cells to exert its cytotoxic effects.

The pharmacokinetics or efficacy of chemotherapeutic agents by microspheres or conventional drug carriers/embolization agents have been compared in several animal studies $(7,8,36)$. One noteworthy study revealed that doxorubicin-loaded HepaSphere has a lower doxorubicin concentration in plasma (within $24 \mathrm{~h}$ ) while a higher concentration in tumor tissue (over 14 days) compared with doxorubicin-loaded lipiodol following TACE treatment in a rabbit model of liver cancer (37). Another study revealed that both doxorubicin and its metabolite doxorubicinol plasma concentrations were lower, whereas the doxorubicin concentration in tumor tissue was higher after being released by DC beads compared with lipiodol in a rabbit model of liver cancer (36). Additionally, in the previous study, it was also revealed that complete necrosis of tumor tissue was achieved after 7 days of doxorubicin-eluting DC bead chemoembolization, but only partial necrosis of tumor tissue was achieved using the doxorubicin/lipiodol emulsion chemoembolization (36). These animal studies revealed that several microspheres are superior to conventional drug carriers/ embolization agents regarding drug pharmacokinetics and treatment efficacy. However, the in vivo ATO pharmacokinetics, efficacy and safety of ATO-eluting CBs are still unclear. Therefore, the ATO pharmacokinetics, tumor necrosis efficacy and safety following chemo-embolizing with ATO-eluting CBs or ATO/lipiodol emulsion in a rabbit VX2 liver cancer model were evaluated. The results revealed that the ATO concentration in plasma was higher, whereas ATO concentration in tumor tissue was higher after chemoembolization of ATO-eluting CBs compared with ATO/ lipiodol emulsion, suggesting that ATO-eluting CBs exhibited improved ATO pharmacokinetics compared with ATO/ lipiodol emulsion. Probable reasons for these results include: i) Lipiodol may circulate with blood, leading to a higher ATO concentration in plasma and a lower ATO concentration in tumor tissue. ii) CBs released ATO in a more sustainable manner compared with lipiodol, which may contribute to its accumulation in tumor tissue and a decreased metabolism speed, thereby increasing its tumor concentration and decreasing its plasma concentration. Additionally, it was revealed that ATO-eluting $\mathrm{CBs}$ enhanced tumor necrosis compared with ATO/lipiodol emulsion at day 7 after the surgery, indicating that ATO-eluting CBs had improved efficacy compared with ATO/lipiodol emulsion (probably due to the higher ATO concentration in tumor tissue after chemoembolizing with ATO-eluting CBs compared with ATO/lipiodol emulsion). Notably, gross observations on liver tissue revealed that ATO-eluting $\mathrm{CBs}$ decreased necrosis of normal liver parenchyma compared with ATO/lipiodol emulsion, which may be due to the fact that ATO/lipiodol emulsion can spread to the normal liver parenchyma, thereby causing necrosis of normal liver parenchyma or lipiodol itself exhibited cytotoxic effects on normal liver cells. However, there was no major difference between soluble and microsphere-loaded ATO in vitro, whereas the use of microspheres resulted in a more sustained release of ATO in the tumor mass (although the initial peak was somewhat lower), the difference of the impact of ATO-eluting CBs on liver cancer between in vivo and in vitro may due to the fact that specifics of release are considerably more complicated in vivo compared with in vitro. Thus, further studies are required in which the tumor cells should initially be incubated in ATO-CBs or a slightly higher concentration of ATO, followed by a wash-out, and subsequently a new incubation of ATO-CBs or ATO at a markedly lower concentration.

In summary, CBs present with acceptable ATO loading and releasing efficiencies. Additionally, ATO-eluting CBs exhibited superior anticancer properties in liver cancer cells, 
and improved pharmacokinetics, treatment efficacy and milder liver toxicity of ATO compared with the ATO/lipiodol emulsion in the TACE treatment of the rabbit model of liver cancer.

\section{Acknowledgements}

Not applicable.

\section{Funding}

The present study was supported by the National Natural Science Foundation of China (grant nos. 81401494 and U2004119).

\section{Availability of data and materials}

The datasets used and analyzed during the current study are available from the corresponding author on reasonable request.

\section{Authors' contributions}

XD and GZ were the main contributors to the research design, and $\mathrm{XH}$ reviewed and revised the study for important intellectual content. In the present study, XD and JR were mainly responsible for the drug loading and release experiments of arsenic trioxide microspheres. XD was mainly responsible for exploration of the dispensing process and dosage of arsenic trioxide loading in drug-carrying microspheres. XH designed the drug loading and release experiments of arsenic trioxide microspheres. JR was mainly responsible for the in vitro experiments assessing the effects of arsenic trioxide microspheres on hepatocellular carcinoma cells. HL was mainly responsible for the establishment of the rabbit VX2 liver tumor model and gradually improved the CT enhanced detection method for the rabbit VX2 liver tumor. PC was mainly responsible for the implementation of TACE treatment in a rabbit VX2 liver tumor model. SJ completed the collection of blood and tissue samples. MW completed the serum (tissue) sample pretreatment and drug concentration detection. SJ and MW jointly completed the analysis and interpretation of the data. In addition, HL, GZ and PC completed the drafting of the manuscipt. All authors read and approved the final manuscript.

\section{Ethics approval and consent to participate}

The study was approved from The Scientific Research and Clinical Trial Ethics Committee of the First Affiliated Hospital of Zhengzhou University (No. Scientific research-2017-03). All the procedures on animals were approved by the Institutional Animal Care and Use Committee at our institution (Ethics and Scientific Trial Committee of the First Affiliated Hospital of Zhengzhou University), and the experiments were performed in accordance with institutional guidelines.

\section{Patient consent for publication}

Not applicable.

\section{Competing interests}

The authors declare that they have no competing interests.

\section{References}

1. Siegel RL, Miller KD and Jemal A: Cancer statistics, 2018. CA Cancer J Clin 68: 7-30, 2018.

2. Bray F, Ferlay J, Soerjomataram I, Siegel RL, Torre LA and Jemal A: Global cancer statistics 2018: GLOBOCAN estimates of incidence and mortality worldwide for 36 cancers in 185 countries. CA Cancer J Clin 68: 394-424, 2018.

3. El-Serag HB: Hepatocellular carcinoma. N Engl J Med 365: 1118-1127, 2011.

4. Jang JH, Lee JW, Hong JT and Jin YJ: Transarterial chemoembolization for hepatocellular carcinoma: An evidence-based review of its place in therapy. J Hepatocell Carcinoma 2: 123-129, 2015.

5. Liu YS, Ou MC, Tsai YS, Lin XZ, Wang CK, Tsai HM and Chuang MT: Transarterial chemoembolization using gelatin sponges or microspheres plus lipiodol-doxorubicin versus doxorubicin-loaded beads for the treatment of hepatocellular carcinoma. Korean J Radiol 16: 125-132, 2015.

6. Xie F, Zang J, Guo X, Xu F, Shen R, Yan L, Yang J and He J: Comparison of transcatheter arterial chemoembolization and microsphere embolization for treatment of unresectable hepatocellular carcinoma: A meta-analysis. J Cancer Res Clin Oncol 138: 455-462, 2012.

7. Zhang S, Huang C, Li Z, Yang Y, Bao T, Chen H, Zou Y and Song L: Comparison of pharmacokinetics and drug release in tissues after transarterial chemoembolization with doxorubicin using diverse lipiodol emulsions and CalliSpheres Beads in rabbit livers. Drug Deliv 24: 1011-1017, 2017.

8. Lee KH, Liapi EA, Cornell C, Reb P, Buijs M, Vossen JA, Ventura VP and Geschwind JF: Doxorubicin-loaded QuadraSphere microspheres: Plasma pharmacokinetics and intratumoral drug concentration in an animal model of liver cancer. Cardiovasc Intervent Radiol 33: 576-582, 2010.

9. Ni JY, Xu LF, Wang WD, Sun HL and Chen YT: Conventional transarterial chemoembolization vs microsphere embolization in hepatocellular carcinoma: A meta-analysis. World J Gastroenterol 20: 17206-17217, 2014.

10. Zhang X, Zhou J, Zhu DD, Huang J, Sun JH, Li TF, Shi CS, Sun ZC, Hou QM, Peng ZY, et al: CalliSpheres(R) drug-eluting beads (DEB) transarterial chemoembolization (TACE) is equally efficient and safe in liver cancer patients with different times of previous conventional TACE treatments: a result from CTILC study. Clin Transl Oncol 21: 167-177, 2019.

11. Yamaguchi T, Seki T, Komemushi A, Suwa K, Tsuda R, Inokuchi R, Murata M, Yuki M, Harima Y and Okazaki K: Acute necrotizing pancreatitis as a fatal complication following DC Bead transcatheter arterial chemoembolization for hepatocellular carcinoma: A case report and review of the literature. Mol Clin Oncol 9: 403-407, 2018.

12. Guan YS, He Q, Jin Y and Yao F: Development of CalliSpheres ${ }^{\circledR}$ embolic microspheres. Zhonghua Gan Zang Bing Za Zhi 24: 549-551, 2016 (In Chinese).

13. Chen G, Zhang D, Ying Y, Wang Z, Tao W, Zhu H, Zhang J and Peng Z: Clinical investigation on transarterial chemoembolization with indigenous drug-eluting beads in treatment of unresectable hepatocellular carcinoma. Zhejiang Da Xue Xue Bao Yi Xue Ban 46: 44-51, 2017 (In Chinese).

14. Wu B, Zhou J, Ling G, Zhu D and Long Q: CalliSpheres drugeluting beads versus lipiodol transarterial chemoembolization in the treatment of hepatocellular carcinoma: A short-term efficacy and safety study. World J Surg Oncol 16: 69, 2018.

15. Zhou GH, Han J, Sun JH, Zhang YL, Zhou TY, Nie CH, Zhu TY, Chen SQ, Wang BQ, Yu ZN, et al: Efficacy and safety profile of drug-eluting beads transarterial chemoembolization by CalliSpheres ${ }^{\circledR}$ beads in Chinese hepatocellular carcinoma patients. BMC Cancer 18: 644, 2018.

16. Cohen MH, Hirschfeld S, Flamm Honig S, Ibrahim A, Johnson JR, O'Leary JJ, White RM, Williams GA and Pazdur R: Drug approval summaries: Arsenic trioxide, tamoxifen citrate, anastrazole, paclitaxel, bexarotene. Oncologist 6: 4-11, 2001.

17. Wang L, Min Z, Wang X, Hu M, Song D, Ren Z, Cheng Y and Wang Y: Arsenic trioxide and sorafenib combination therapy for human hepatocellular carcinoma functions via up-regulation of TNF-related apoptosis-inducing ligand. Oncol Lett 16: 3341-3350, 2018.

18. Zhang F, Zhang CM, Li S, Wang KK, Guo BB, Fu Y, Liu LY, Zhang Y, Jiang HY and Wu CJ: Low dosage of arsenic trioxide inhibits vasculogenic mimicry in hepatoblastoma without cell apoptosis. Mol Med Rep 17: 1573-1582, 2018. 
19. Wang HY, Zhang B, Zhou JN, Wang DX, Xu YC, Zeng Q, Jia YL, $\mathrm{Xi} J \mathrm{~J}, \mathrm{Nan} \mathrm{X}, \mathrm{He} \mathrm{LJ}$, et al: Arsenic trioxide inhibits liver cancer stem cells and metastasis by targeting SRF/MCM7 complex. Cell Death Dis 10: 453, 2019.

20. Qiu Y, Dai Y, Zhang C, Yang Y, Jin M, Shan W, Shen J, Lu M, Tang Z, Ju L, et al: Arsenic trioxide reverses the chemoresistance in hepatocellular carcinoma: A targeted intervention of 14-3-3eta/NF-kappaB feedback loop. J Exp Clin Cancer Res 37: 321, 2018.

21. Song P, Hai Y, Ma W, Zhao L, Wang X, Xie Q, Li Y, Wu Z, $\mathrm{Li} \mathrm{Y}$ and Li H: Arsenic trioxide combined with transarterial chemoembolization for unresectable primary hepatic carcinoma: A systematic review and meta-analysis. Medicine (Baltimore) 97: e0613, 2018

22. Schiavone EL and Torrado OA: Determination of arsenic in water by the silver diethyldithiocarbamate method. Rev Sanid Milit Argent 66: 251-263, 1967 (In Spanish).

23. Livak KJ and Schmittgen TD: Analysis of relative gene expression data using real-time quantitative PCR and the 2(-Delta Delta C(T)) Method. Methods 25: 402-408, 2001

24. Cima G: AVMA Guidelines for the Euthanasia of Animal. Javma Journal of the American Veterinary Medical Association. 2013 edition. 242: 715-716, 2013.

25. Sharma BK, Srinivasan R, Chawla YK and Chakraborti A Vascular endothelial growth factor: Evidence for autocrine signaling in hepatocellular carcinoma cell lines affecting invasion. Indian J Cancer 53: 542-547, 2016.

26. Zhou L, Wang DS, Li QJ, Sun W, Zhang Y and Dou KF Downregulation of the Notch signaling pathway inhibits hepatocellular carcinoma cell invasion by inactivation of matrix metalloproteinase-2 and -9 and vascular endothelial growth factor. Oncol Rep 28: 874-882, 2012.

27. Nouri YM, Kim JH, Yoon HK, Ko HK, Shin JH and Gwon DI: Update on transarterial chemoembolization with drug-eluting microspheres for hepatocellular carcinoma. Korean J Radiol 20: 34-49, 2019.

28. Huang $\mathrm{K}$, Zhou Q, Wang $\mathrm{R}$, Cheng $\mathrm{D}$ and Ma Y: Doxorubicin-eluting beads versus conventional transarterial chemoembolization for the treatment of hepatocellular carcinoma. J Gastroenterol Hepatol 29: 920-925, 2014.
29. Zhang X, Jia S, Yang S, Yang Y, Yang T and Yang Y: Arsenic trioxide induces G2/M arrest in hepatocellular carcinoma cells by increasing the tumor suppressor PTEN expression. J Cell Biochem 113: 3528-3535, 2012

30. Jiang L, Wang L, Chen L, Cai GH, Ren QY, Chen JZ, Shi HJ and Xie YH: As2O3 induces apoptosis in human hepatocellular carcinoma HepG2 cells through a ROS-mediated mitochondrial pathway and activation of caspases. Int J Clin Exp Med 8: 2190-2196, 2015.

31. Cui L, GaoB,CaoZ, Chen X,Zhang S andZhang W: Downregulation of B7-H4 in the MHCC97-H hepatocellular carcinoma cell line by arsenic trioxide. Mol Med Rep 13: 2032-2038, 2016.

32. Yu H, Zhu GY, Xu RZ, Niu HZ, Lu Q, Li GZ, Wang ZY, Zhang DS, Gu N and Teng GJ: Arterial embolization hyperthermia using $\mathrm{As}_{2} \mathrm{O}_{3}$ nanoparticles in $\mathrm{VX}_{2}$ carcinoma-induced liver tumors. PLoS One 6: e17926, 2011.

33. Tan B, Huang JF, Wei Q, Zhang H and Ni RZ: Anti-hepatoma effect of arsenic trioxide on experimental liver cancer induced by 2 -acetamidofluorene in rats. World J Gastroenterol 11: 5938-5943, 2005.

34. Jordan O, Denys A, De Baere T, Boulens N and Doelker E: Comparative study of chemoembolization loadable beads: In vitro drug release and physical properties of DC bead and hepasphere loaded with doxorubicin and irinotecan. J Vasc Interv Radiol 21: 1084-1090, 2010.

35. de Baere T, Plotkin S, Yu R, Sutter A, Wu Y and Cruise GM: An in vitro evaluation of four types of Drug-eluting microspheres loaded with doxorubicin. J Vasc Interv Radiol 27: 1425-1431, 2016.

36. Hong K, Khwaja A, Liapi E, Torbenson MS, Georgiades CS and Geschwind JF: New intra-arterial drug delivery system for the treatment of liver cancer: preclinical assessment in a rabbit model of liver cancer. Clin Cancer Res 12: 2563-2567, 2006.

37. Gupta S, Wright KC, Ensor J, Van Pelt CS, Dixon KA and Kundra V: Hepatic arterial embolization with doxorubicinloaded superabsorbent polymer microspheres in a rabbit liver tumor model. Cardiovasc Intervent Radiol 34: 1021-1030, 2011.

(i) $($ ) This work is licensed under a Creative Commons Attribution-NonCommercial-NoDerivatives 4.0 International (CC BY-NC-ND 4.0) License. 